\title{
ENTIRE FUNCTIONS OF BOUNDED INDEX
}

\author{
FRED GROSS
}

I. Introduction. An entire function, $f(z)$, has a Taylor expansion about any point $a$ in the complex plane of the form

$$
f(z)=\sum_{i=0}^{\infty} a_{n}(z-a)^{n} .
$$

Since this series is absolutely convergent everywhere in the plane, the terms $\left|a_{n}\right|$ must approach 0. Consequently, there exists for each $a$, an index $n_{0}=n(a)$ for which $\left|a_{n}\right|$ is a maximal coefficient. B. Lepson [2] raised the problem of characterizing entire functions for which $n(a)$ is bounded. The latter are called functions of bounded index. In what follows, we shall give a partial solution to Lepson's problem. We shall also include a number of results using somewhat different conditions than those suggested by Lepson.

II. Functions of bounded index and nonuniform bounded index.

Definition 1. An entire function is said to be of bounded index if and only if there exists an integer $N$, such that for all $z$

$$
\max \left(|f|,\left|f^{(1)}\right|, \frac{\left|f^{(2)}\right|}{2 !}, \cdots, \frac{\left|f^{(N)}\right|}{V !}\right) \geqq \frac{\left|f^{(j)}\right|}{j !} ;
$$

$\left(f^{(0)} \operatorname{denotes} f\right)$. We shall say that $f$ is of index $N$, if $N$ is the smallest integer for which (1) holds. An entire function which is not of bounded index is said to be of unbounded index.

A function of bounded index satisfies

$$
\sum_{i=0}^{N} \frac{\left|f^{(i)}\right|}{i !} \geqq \frac{\left|f^{(j)}\right|}{j !} ; \quad j=0,1,2,3, \cdots
$$

Furthermore, if (2) holds then

$$
\begin{aligned}
& \max \left(|f|,\left|f^{(1)}\right|, \frac{\left|f^{(2)}\right|}{2 !}, \cdots, \frac{f^{(N)}}{N !}\right) \geqq \frac{1}{(N+1)} \frac{\left|f^{(j)}\right|}{j !} \\
& \quad j=0,1,2,3, \cdots .
\end{aligned}
$$

These facts suggest

Definition 2. An entire function $f(z)$ is said to be of nonuniform

\footnotetext{
Received by the editors September 30, 1966.
} 
bounded index if and only if there exist integers $N_{j}$, such that

$$
\sum_{i=0}^{N} \frac{\left|f^{(i)}(z)\right|}{i !} \geqq c \frac{\left|f^{(j)}(z)\right|}{j !} \text { for }|z|>N_{j} ; \quad j=0,1,2,3, \ldots
$$

$c$ is any fixed constant.

For bounded regions Lepson [2] proved that: If $f(z) \not \equiv 0$ is an entire function and $R$ is any bounded set, then there is an integer $N$, such that for any $z$ in $R$ and any nonnegative integer $n$

$$
\frac{\left|f^{(n)}(z)\right|}{n !} \leqq \max \left(|f(z)|,\left|f^{(1)}(z)\right|, \frac{\left|f^{(2)}(z)\right|}{2 !}, \cdots, \frac{\left|f^{(N)}(z)\right|}{N !}\right) .
$$

The function $e^{z}$ is obviously of bounded index. More generally, we prove the simple

TheOREM 1. If $f$ is entire and satisfies $f^{(k+1)}=f$, then it is of bounded index.

Proof. Write $j=q(k+1)+r, r \leqq k$. Then

$$
\begin{aligned}
\left|\frac{f^{(j)}}{j !}\right| & =\left|\frac{f^{q(k+1)+r}}{(q(k+1)+r) !}\right|=\left|\frac{f^{(r)}}{(q(k+1)+r) !}\right|<\left|\frac{f^{(r)}}{r !}\right| \\
& \leqq \max \left(|f|,\left|f^{(1)}\right|, \cdots,\left|\frac{f^{(k)}}{k !}\right|\right)
\end{aligned}
$$

and Theorem 1 follows.

As another generalization of the case $f(z)=e^{z}$ we prove:

Theorem 2. $f=Q e^{a z}$ is of bounded index whenever $Q$ is a polynomial.

Proof. We may assume without any loss of generality that the leading coefficient of $Q$ is 1 . Let

$$
p_{k}=\frac{f^{(k)}}{e^{a z}}=\sum_{i=0}^{n} c_{i}^{k} Q^{(i)} a^{k-i} ; \quad c_{i}^{k}=\frac{k(k-1) \cdots(k-i+1)}{i !}<k^{n}
$$

for $1 \leqq i \leqq k ; c_{0}^{k}=1$ and $c_{i}^{k}=0$ for $i>k$, where $n$ is the degree of $Q$.

$\left|Q^{(i)}\right|<3 r^{n} / 2$ for all nonnegative integers $i$ and for sufficiently large $r$; say $r>r_{0}$.

Assume that $f$ is of unbounded index. Then there exist an infinite sequence of $k$ and a corresponding sequence $z_{k}$ both going to $\infty$ such that

$$
\left|f\left(z_{k}\right)\right|<\left|f^{k}\left(z_{k}\right)\right| / k !
$$

This implies that 


$$
\left|Q\left(z_{k}\right)\right|<\sum_{i=0}^{n} \frac{\left|c_{i}^{k} Q^{(i)}\left(z_{k}\right) a^{k-i}\right|}{k !} .
$$

Hence, it suffices to show that (3) is impossible when $|a|>1$, i.e., to show that we cannot have

$$
\left|Q\left(z_{k}\right)\right|<\sum_{i=0}^{n} \frac{\left|c_{i}^{k}\right|\left|Q^{(i)}\left(z_{k}\right)\right||a|^{k}}{k !}<\frac{3}{2} \frac{k^{n} r_{k}^{n}|a|^{k}}{k !},
$$

where $r_{k}=\left|z_{k}\right|$.

Since $|Q|>r^{n} / 2$ for sufficiently large $r$, say $r>r_{1},\left(r_{1}\right.$ independent of $k$, of course) it follows that (4) would imply that

$$
1<3 k^{n}|a|^{k} / k \text { ! }
$$

for an infinite sequence of $k \rightarrow \infty$. Since (5) is impossible our theorem follows.

One might conjecture that $f(z)$ is of bounded index if and only if $f\left(z^{k}\right)$ is, where $k$ is a positive integer. This, however, is false, as illustrated by the pair $e^{z}$ and $e^{z^{2}}$; since $e^{z}$ is of bounded index and $e^{z^{2}}$ is not, as we shall see in the next section. Nevertheless one can prove the following:

THEOREM 3. Let $t$ be a positive integer. If $g(z)=f\left(z^{1 / t}\right)$ is entire and $f(z)$ is of bounded index (say of index $k$ ) then $g(z)$ is of nonuniform bounded index.

Proof. We may assume that $t>1$. Using mathematical induction and performing some elementary calculations one can verify that for any positive integer $n$

$$
g^{(n)}(z)=p_{1 n} f^{(1)}\left(z^{1 / t}\right)+p_{2 n} f^{(2)}\left(z^{1 / t}\right)+\cdots+p_{n n} f^{(n)}\left(z^{1 / t}\right),
$$

where $p_{i n}=c_{\text {in }}\left(z^{-1 / t}\right)^{n t-i}, c_{\text {in }}$ a nonzero constant; $n=1,2,3, \cdots$.

Using (6) one shows easily that

$$
\begin{aligned}
& |g(z)|+\left|g^{\prime}(z)\right|+\frac{\left|g^{(2)}(z)\right|}{2 !}+\cdots+\frac{\left|g^{(k)}(z)\right|}{k !} \\
& \quad \geqq\left|f\left(z^{1 / t}\right)\right|+\left(\left|p_{11}^{*}\right|-\sum_{j=2}^{k}\left|p_{1 j}^{*}\right|\right)\left|f^{(1)}\left(z^{1 / t}\right)\right| \\
& \quad+\left(\left|p_{22}^{*}\right|-\sum_{j=3}^{k}\left|p_{2 j}^{*}\right|\right) \frac{\left|f^{(2)}\left(z^{1 / t}\right)\right|}{2 !}+\cdots \\
& \quad+\left(\left|p_{i i}^{*}\right|-\sum_{j=i+1}^{k}\left|p_{i j}^{*}\right|\right) \frac{\left|f^{(i)}\left(z^{1 / t}\right)\right|}{i !}+\cdots+\left|p_{k k}^{*}\right| \frac{\left|f^{(k)}\left(z^{1 / t}\right)\right|}{k !} .
\end{aligned}
$$


The $p_{i j}{ }^{*}$ 's are the same as the $p_{i j}$ 's except for some positive constant multipliers.

On the other hand, for any integer $s>k$

$$
\frac{\left|g^{(s)}(z)\right|}{s !} \leqq \sum_{i=1}^{s}\left|p_{i s}^{*}\right| \frac{\left|f^{(i)}\left(z^{1 / t}\right)\right|}{i !} .
$$

Thus to prove Theorem 3 it suffices to show that

$$
\begin{aligned}
|f| & +\left(\left|p_{11}^{*}\right|-\sum_{j=2}^{k}\left|p_{1 j}^{*}\right|-\left|p_{1 s}^{*}\right|\right)\left|f^{(1)}\right| \\
& +\left(\left|p_{22}^{*}\right|-\sum_{j=3}^{k}\left|p_{2 j}^{*}\right|-\left|p_{2 s}^{*}\right|\right) \frac{\left|f^{(2)}\right|}{2 !}+\cdots \\
& +\left(\left|p_{i i}^{*}\right|-\sum_{j=i+1}^{k}\left|p_{i j}^{*}\right|\right) \frac{\left|f^{(i)}\right|}{i !}+\cdots \\
& +\left(\left|p_{k k}^{*}\right|-\left|p_{k s}^{*}\right|\right) \frac{\left|f^{(k)}\right|}{k !} \\
> & \left|p_{(k+1) s}^{*}\right| \frac{\left|f^{(k+1)}\right|}{(k+1) !}+\left|p_{(k+2) s}^{*}\right| \frac{\left|f^{(k+2)}\right|}{(k+2) !}+\cdots+\left|p_{s s}^{*}\right| \frac{\left|f^{(s)}\right|}{s !}
\end{aligned}
$$

for sufficiently large $|z|=r$.

The terms $\left|p_{i i}^{*}\right|$ dominate the coefficients of $\left|f^{(i)}\right|$ (which we denote by $c_{i}$ ), since they have minimal degree (in the variable $z^{-1 / t}$ ) in each term. Using this fact one can easily show that for any $c>0$

$$
\frac{1}{s} \sum_{i=0}^{k}\left|\frac{c_{i}}{p_{(k+j)_{s}}^{*}}\right| \frac{\left|f^{(i)}\right|}{i !}>c \sum_{i=1}^{k} \frac{\left|f^{(i)}\right|}{i !}>c \frac{\left|f^{(k+j)}\right|}{(k+j) !}
$$

for $j=1,2, \cdots, s-k$, and $|z|>N(s) ; N(s)$ some integer dependent on $s$. The last inequality follows from the hypotheses of our theorem.

From (10) we get

$$
s\left(\frac{1}{s} \sum_{i=0}^{k}\left|c_{i}\right| \frac{\left|f^{(i)}\right|}{i !}\right)>c \sum_{j=1}^{s-k}\left|\stackrel{*}{p_{(k+j) s} \mid}\right| \frac{\left|f^{(k+j)}\right|}{(k+j) !}
$$

for $|z|>N(s)$ and our proof is complete.

As an application of Theorem 3 we have

Corollary. Let $k$ be a positive integer. The entire function

$$
f(z)=\sum_{n=0}^{\infty} \frac{z^{n}}{(k n) !}
$$

is of nonuniform bounded index. 
Proof. $f(z)$ can be expressed as

$$
f(z)=\sum_{n=0}^{\infty} \frac{\left(z^{1 / k}\right)^{k_{n}}}{(k n) !}=g\left(z^{1 / k}\right),
$$

where $g(z)$ satisfies $g^{(k)}(z)=g(z)$. Thus, by virtue of Theorem $1, g(z)$ is of bounded index. Hence, by Theorem 3, $f(z)$ is of nonuniform bounded index.

It is very likely that Theorem 3 can be generalized to the following

CONJECTURE. If $g(z)$ is of unbounded index and $p(z)$ is a polynomial then $g(p(z))$ is also of unbounded index.

III. Functions of unbounded index. We begin with the following lemma due to E. Borel [1].

LEMMA 1. If $V(r)$ is monotonic increasing, then for any $\epsilon^{\prime}>0$ and $\epsilon>0$

$$
V\left(r+\frac{1}{(\log V(r))^{1+\epsilon^{\prime}}}\right) \leqq(1+\epsilon) V(r)
$$

for all $r$ outside a set of finite measure.

Proof. See Hayman [1, Lemma 2.4 (i), pp. 38-39], for the proof of a similar result.

Lemma 2. For any entire function $f(z)$ and any $\epsilon>0$

$$
M_{f^{(n)}}(r) \leqq M_{f}(r)^{1+\epsilon}
$$

for all $r$ outside a set of finite measure. This exceptional set may depend on $n$.

Proof. It is well known (see [3]) that for $R>r$

$$
M_{f^{\prime}}(r) \leqq \frac{M_{f}(R)}{R-r} \cdot\left(M_{f}(r) \text { denotes } \max _{|z|=r}|f(z)|\right) .
$$

Choose

$$
R=r+\frac{1}{\left(\log M_{f}(r)\right)^{1+\epsilon^{\prime}}} ; \quad \epsilon^{\prime}>0 .
$$

We get by virtue of Lemma 1 that

$$
\begin{aligned}
M_{f^{\prime}}(r) & \leqq M_{f}\left(r+\frac{1}{\left(\log M_{f}(r)\right)^{1+\epsilon^{\prime}}}\right) \cdot\left(\log M_{f}(r)\right)^{1+\epsilon^{\prime}} \\
& <(1+\epsilon) M_{f}(r)\left(\log M_{f}(r)\right)^{1+\epsilon^{\prime}}
\end{aligned}
$$


for all $r$ outside a set of finite measure.

Thus the assertion of our lemma is valid when $n=1$.

Suppose that $M_{f^{(k)}}(r) \leqq M_{f}(r)^{1+\epsilon}$. Then by (11)

$$
\begin{aligned}
M_{f^{(k+1)}}(r) & \leqq(1+\epsilon) M_{f^{(k)}}(r)\left(\log M_{f^{(k)}}(r)\right)^{1+\epsilon^{\prime}} \\
& \leqq(1+\epsilon)^{3} M_{f}(r)^{1+\epsilon}\left(\log M_{f}(r)\right)^{1+\epsilon^{\prime}}<M_{f}(r)^{1+\epsilon^{\prime \prime}} \quad\left(\epsilon^{\prime \prime}>\epsilon\right)
\end{aligned}
$$

for all $r$ outside a set of finite measure. Our lemma follows.

We now list some additional obvious properties of the maximum modulus function that we shall use in the sequel:

(a) $M_{f g}(r) \leqq M_{f}(r) M_{g}(r)$.

(b) $M_{f^{n}}(r)=M_{f}(r)^{n}$.

(c) If $M_{g}(r)<\epsilon M_{f}(r)$ for sufficiently large $r$, then for any $\epsilon>0$, $(1-\epsilon) M_{f}(r)<M_{f+g}(r)<(1+\epsilon) M_{f}(r)$ for sufficiently large $r$.

(d) If $f(z)$ is transcendental, then for any $\epsilon>0, M_{f}(r)<M_{f^{\prime}}(r)^{1+\epsilon}$ for sufficiently large $r$.

THEOREM 4. Let $\alpha$ and $\phi$ be any two transcendental entire functions and let $\delta$ be a real number less than $\frac{1}{2}$. If

$$
M_{\alpha}(r)<M_{\phi}(r)^{\delta}
$$

for a set, $s$, of $r$ of infinite measure, then $f=\alpha e^{\phi}$ is of unbounded index.

If $\alpha$ is a polynomial and $\phi$ is entire then $f$ is of unbounded index.

Proof. We prove the first part of the theorem. The second part follows from a similar argument. It follows by mathematical induction that

$$
\begin{aligned}
f^{(n)} & =\left[\left(\phi^{\prime}\right)^{n} \alpha+p_{n}\left(\phi^{\prime}, \phi^{(2)}, \cdots, \phi^{(n)}, \alpha, \alpha^{\prime}, \cdots, \alpha^{(n)}\right)\right] e^{\phi} \\
& =h_{n} e^{\phi}
\end{aligned}
$$

where $p_{n}$ is a polynomial in $\phi^{\prime}, \phi^{(2)}, \cdots, \alpha, \alpha^{\prime}, \cdots, \alpha^{(n)}$ whose degree in the $\phi$ 's is less than or equal to $n-1$ and where at most one of $\alpha, \alpha^{\prime}, \cdots, \alpha^{(n)}$ appears in any one term.

It follows from (12) and the properties of the maximum modulus function that for any $\epsilon>0$

$$
M_{p_{n}}(r) \leqq M_{\phi^{\prime}}(r)^{(n-1)(1+\epsilon)+(1+\epsilon) \delta}
$$

for all $r$ in the set $s$, with the possible exception of a set of finite measure. Choosing $\epsilon$ sufficiently small, we deduce from (12) that

$$
\begin{aligned}
\left(M_{\phi^{\prime}}(\boldsymbol{r})\right)^{n-\delta}-( & \left.M_{\phi^{\prime}}(\boldsymbol{r})\right)^{(n-1)(1+\epsilon)+\delta(1+\epsilon)}-M_{h_{n}}(\boldsymbol{r}) \\
& <M_{\phi^{\prime}}(\boldsymbol{r})^{n+\delta}+M_{\phi^{\prime}}(\boldsymbol{r})^{(n-1)(1+\epsilon)+(1+\epsilon) \delta}
\end{aligned}
$$

or for sufficiently small $\epsilon$ we have 


$$
(1-\epsilon)\left(M_{\phi^{\prime}}(r)\right)^{n-\delta}-M_{h_{n}}(r)<(1+\epsilon)\left(M_{\phi^{\prime}}(r)\right)^{n+\delta}
$$

for a set of $r$ of infinite measure.

Now assume that $f$ is of index $k$; then

$$
|f|+\left|f^{\prime}\right|+\frac{\left|f^{(2)}\right|}{2 !}+\cdots+\frac{\left|f^{(k)}\right|}{k !}>\frac{\left|f^{(m)}\right|}{m !} \quad(m>k) .
$$

Hence, from (12) we find that

$$
|\alpha|+\left|h_{1}\right|+\frac{\left|h_{2}\right|}{2 !}+\cdots+\frac{\left|h_{k}\right|}{k !}>\frac{\left|h_{m}\right|}{m !}
$$

and consequently

$$
M_{\alpha}(r)+M_{h_{1}}(r)+\frac{M_{h_{2}}(r)}{2 !}+\cdots+\frac{M_{h_{k}}(r)}{k !}>\frac{M_{h_{m}}(r)}{m !} .
$$

From (13) and (15) we get for sufficiently small $\epsilon_{0}>0$

$$
M_{\alpha}(r)+M_{h_{1}}(r)+\frac{M_{h_{2}}(r)}{2 !}+\cdots+\frac{M_{h_{k}}(r)}{k !}<\left(M_{\phi^{\prime}}(r)\right)^{k+\delta+\epsilon_{0}}
$$

for a set, $s^{\prime}$, of $r$ of infinite measure. On the other hand

$$
M_{h_{m}}(r) / m !>\left(M_{\phi^{\prime}}(r)\right)^{m-\delta-\epsilon_{0}}
$$

for a subset of $s^{\prime}$ of infinite measure.

Since (16) and (17) contradict (15) our theorem follows.

Corollary. An entire function with at most a finite number of zeros is of bounded index if and only if it is of the form $p(z) e^{a z}$, where $p(z)$ is polynomial and $a$ is a complex constant.

It is worth noting that the proof of Theorem 4 can be used to generalize the results of that theorem to functions of the form $\alpha e^{\phi}+\beta$ provided that suitable growth restrictions are imposed on $\beta$.

The methods of this paper are elementary. One can probably get somewhat stronger results by using the Wiman-Valiron theory.

\section{REFERENCES}

1. W. K. Hayman, Meromorphic functions, Oxford Math. Monographs, Clarendon Press, Oxford, 1964.

2. B. Lepson, Differential equations of infinite order, hyperdirichlet series and entire functions of bounded index, Lecture Notes, 1966, Summer Institute on Entire Functions, Lniv. of California, La Jolla, Calif.

3. E. C. Titchmarsh, The theory of functions, 2nd ed., Oxford Univ. Press, Oxford, 1939.

Bellcomm InC., Washington, D. C. 